\title{
High Platelet Lymphocyte Ratio as Predictor of Bad Outcome in Patient with Acute Ischemic Stroke
}

\author{
Fani Nanda Sihanto ${ }^{1}$, Mochamad Bahrudin ${ }^{1}$, Suharto $^{1}$ \\ ${ }^{1}$ Faculty of Medicine, Muhammadiyah Malang University, Malang, East Java, Indonesia \\ Corresponding Author: Fani Nanda Sihanto
}

\begin{abstract}
Background/Aim: Stroke is a major cause of death and disability worldwide. Inflammatory markers for poor outcomes in stroke patients have been widely studied, but these examinations are often constrained by limited costs and facilities. Platelet lymphocyte ratio is one of the markers of inflammation that is easily examined, only a few researches were studied on stroke patients, especially ischemic type. This article aimed to understanding high platelet lymphocyte ratio as a risk factor for poor outcome in acute ischemic stroke patients.

Method: The design of this research is literature review by literature from 32 journal and 5 textbooks. Journals obtained from search engines Pubmed, Google Scholar, and NCBI.

Result: Based on the results of the literature review, circulating platelets play an important role in the development and resolution, not only because of their direct effect on the endothelium but also by releasing inflammatory mediators. Acute ischemic stroke will induce lymphocyte apoptosis, which causes impaired immune system. High platelet counts and low lymphocytes contribute to micro-thrombus formation, which increases the risk of stroke and has a poor prognosis in patient.

Conclusion: There is an increase platelet lymphocyte ratio can be an independent predictor of poor outcome in acute ischemic stroke patient
\end{abstract}

Keywords: acute ischemic, stroke, platelet lymphocyte ratio, poor outcome

\section{INTRODUCTION}

Stroke according to World Health Organization (WHO) is a sign focal or global clinical that happened sudden, annoying cerebral function, and lasts for more than 24 hours or cause death, without cause other than vascular. Stroke divided into two groups big, that is stroke ischemia and hemorrhagic.[1] Stroke is a disease dead cause number two in world and causes number defect three in the world. In America United, there are 795,000 cases in a year that divided into 610,000 stroke cases first time and 185,000 happens to the patient who have experienced previous stroke, as well as case death 140,000 per years.[2] In Asia, the incident stroke is 116483/100,000 inhabitants per year.[3] In Indonesia number of sufferers stroke in 2018 as much 713,783 cases, and in Java East is approach 113,045 cases.[4]

Management patients with continuous stroke developed to treatment that fast and precise on target can reduce morbidity rate in patients with strokes. In patients with treatment late or not usually right will cause treatment and the treatment takes a lot of time relatively long so can cause social problems in public.[2]

Biomarkers required

for determination early treatment strokes. Biomarkers good can be as ischemic stroke marker acute, is that which reaches peak and accumulate at the start of the cascade from ischemic processes, has fast diffusion process via network ischemia to blood flow. Information obtained from the results biomarkers and imaging results neuroimaging on acute phase can give you 
an idea salvageable network and can provide therapy more precise.[5]

One of factors that play a role in the pathogenesis of stroke is inflammation. Inflammation is response to injury on living tissue have vascularity.[6] Biomarkers as inflammatory markers, wrong only Platelet Ratio Lymphocytes (RTL). RTL is wrong one marker cheap inflammation and easy to check, however still rarely researched in stroke, in particular ischemic stroke.[7]

Enhancement RLT can also used as predictor long term mortality and no only on condition patients of stroke. Platelets secrete thromboxane and mediator other so increase in platelets will cause increased inflammation.[8] Lymphocytes is a subtype from the leukocyte group, which is also take part in the process inflammation. Lymphocytes show a neuroprotective effect, i.e. lymphocytes $\mathrm{T}$ regulatory (Treg), where This Treg will secrete anti-inflammatory cytokines such as IL-10 and tumors growth factor- $\beta$ (TGF- $\beta)$ which will suppress cytokines pro-inflammatory IL-6 and TNF- $\alpha$. Decrease lymphocyte count will be lead to response prolonged inflammation and influence stroke output. So that High RTL accompanied by risk factors conventional can used as a marker in predicting stroke.[9]

\section{METHOD}

Method good literature search national and international done through NCBI, Pubmed, and Google Scholar. Say used key including stroke acute ischemia, platelet ratio with lymphocytes, and bad outside. Obtained library will then be read, analyzed and then discussed. Journal limitation that is published Last 5 years and appropriate by topic that was lifted. Pick up library of 32 journals with details of 20 Q1 and 11 indexed journals Q2 indexed journal, 1 journal indexed $\mathrm{S} 2$ on Sinta Indonesian, and 5 textbook.

\section{RESULT AND DISCUSSION}

Stroke ischemia can occur because of a the process that started by the presence of a thrombus or embolism in the blood vessels cause disturbance metabolism in cells brain, caused by blood supply, oxygen and disturbed energy. Stroke ischemic will trigger reaction inflammation in the area affected, who will experience the progression of the day up to a few weeks after the appearance of symptoms.

The inflammatory process contributes to progression of injury ischemic brain, which exacerbates from tissue damage and exacerbation of deficit neurological. Ischemia cerebral has relationship with infiltration from inflammatory cells who entered into the territory ischemic.[10]

Process inflammation through several studies proved to be a factor important in regeneration or brain damage. Inflammation level high causes brain damage after ischemic process or can be as breadth marker brain damage that happened consequence strokes. Bookmark inflammation that evaluated like thrombocytes, lymphocytes, and RTL.

Study meta-analysis in year 2019 get on patients with stroke acute ischemia has higher platelet count low when compared with normal people, as determined by decrease significantly aggregation level the platelets induced by adenosine diphosphate (ADP). Platelets lower on acute ischemic stroke can contribute on platelet consumption during the acute phase of stroke.[11] It is supported by 2019 research on patient with thrombocytopenia $(<100,000 / \mathrm{mm} 3)$ comorbid related with more mortality height and length of stay longer stay at the hospital and pose a risk of bleeding the intracranial higher. The result is presumed caused by increased consumption platelets in the process platelet activation and atherothrombotic process sustainable will cause count decreased platelets. A little different from research in the year of 2019, get high platelet levels means as risk factors bad outside.[7] Study this also get platelet levels $249-450 \times 109 / \mu \mathrm{L}$ has relationship 
with output poor clinical course, including stroke repetition, and motility.[12] So, circulating platelets play an important role in development and stroke resolution ischemic, not only because of the direct effect on the endothelium but also by acting as a mediator for cells other circulation by facilitating activation and release the activator saved in details platelets.

When ischemic stroke acute occurs, platelet function abnormal, and then activation and accumulation excessive platelets can cause thrombosis and vascular obstruction, which in turn cause incident vascular. Stress during ischemic stroke incidence acute cause Activation hypothalamus-pituitary-adrenal (HPA) axis, so that can increase secretion cortisol causes concentration drop lymphocytes. [13] Study 2019 retrospective shows median lymphocyte levels in bad outside $1,1 \times 103 / \mu \mathrm{L}$ lower compared to the outside is $\operatorname{good} 1.5 \times 103 / \mu \mathrm{L} 14$. That matter supported on research 2021 concludes lymphopenia in patients acute ischemic stroke is a marker of decreased response immunity such as decreased cell count natural killer (NK), B cells, and $\mathrm{T}$ cells in peripheral blood and spleen so susceptible to bacterial infections, as well as can cause brain damage heavier, so result in the poor outside.[7]

Study on 2019 shows that platelets and lymphocytes are predictor of prognosis on disease ischemic vasculature, especially myocardial infarction and cerebral infarction. RTL is biochemical factors for predict prognosis acute ischemic stroke. RTL calculated with the sum formula platelets / count lymphocytes.[7] In a 2016 study of cerebrovascular disease, an increase in RTL was considered a predictor of stroke.[14] This is supported by research in 2017 showing that a low RTL level can be a very high predictive value negative to rule out the incidence of stroke in patients with arterial stenosis karotis.[15] In the research in 2019 obtained RTL 128 has correlation with clinical outcome of patients with acute ischemic stroke. More RTL high in the case group can be suspected because RTL is a systemic inflammatory biomarkers correlated with functional outcome Stroke sufferers, acute ischemic.[16] In research, it also shows Increased $\hat{R T L}$ in patients with acute ischemic stroke with NIHSS 6 when compared ischemic stroke patients acute with NIHSS < 6 (point value cut RTL 128) with $50 \%$ sensitivity and $81 \%$ specificity so that RTL̂ is said to be good as a predictor of a degree output severity of stroke. Research in 2016 shows that RTL >145 increase risk of death 4 to 5 times. The mortality rate was significantly higher in patients with RTL >145 if compared to with RTL $<145$.[13] Both results The study showed similar results, this could indicate that RTL as biomarkers for show the outside worse than acute ischemic stroke.

In the 2019 study, we found cases with a history of infection and lymphocytopenia can improve susceptibility to stroke.[17] With mechanism local inflammation of the brain parenchyma and meninges, through inflammation systemic by promoting atherosclerosis thereby causing coagulation and dysfunction note, and can also directly induce schemia. In the study, also patients with immunocompromised show enhancement risk bad output in ischemic stroke, about three times fold. It shows the immune system has a neuroprotective effect. If the patient has lymphocytopenia, the effects of neuroprotective will be reduced resulting in damage will not increase. Supported Also with research in 2019 showed that patients who were immunocompromised due to systemic infection increases cerebrovascular disease risk and provide a poor prognosis.[18] The two research results can indicate that RTL as a biomarker can be used in patients with immunocompromised or lymphocytopenia previously for Assessing poor outcome in patients with acute ischemic stroke.

From several discussions regarding the relationship between RTL and acute 
ischemic stroke, it was found that the link between the two is the pathological process of acute ischemic stroke which affect on inflammatory reaction. Platelets have an important role in the formation of atherosclerosis, by playing a role in plaque formation and fibrin network formation as a result of rupture plaque which is unstable. Platelets can be inflammatory mediators like chemokines and Cytokines. Lymphocytes have important role of subtypes a family of leukocytes, which play a role in an inflammatory process. From The discussion shows the existence of immune system dysfunction after ischemia cerebral. Ischemia cerebral will induces lymphocyte apoptosis. Cerebral ischemia induces apoptotic process, extensive lymphocytes and quickly in organs, lymphoid and blood. This appears 6-12 hours after cerebral ischemia so that it will cause decrease in blood lymphocytes. Increased levels of RTL also represent formation of micro-thrombus, which will increase the risk of stroke and having poor prognostic association in patients with ischemic stroke.

\section{CONCLUSION}

Based on the theory of discussion carried out, it can be concluded that high RTL is independent risk factor bad output during care for ischemic stroke patients. Patients with acute ischemic stroke with High RTL has high risk to experience bad output during treatment compared patients with acute ischemic stroke with low RTL.

\section{Funding}

This work did not receive any grant from funding agencies in the public, commercial, or not-for-profit sectors.

\section{Conflict Of Interest}

There are no conflicts of interest to declare by any of the authors of this study. Acknowledgement: None

\section{REFERENCES}

1. Coupland AP, Thapar A, Qureshi MI, Jenkins H, Davies AH. The definition of stroke. J R Soc Med. 2017;110(1):9-12.

2. Campbell BCV, Khatri P. Stroke. Lancet. 2020;396(10244):129-42.

3. Venketasubramanian N, Yoon W, Pandian J, Navarro C. Stroke Epidemiology in South , East, and South-East Asia: A Review. 2017;19(3):286-94.

4. Fitri FI, Rambe AS, Fithrie A. The Profile of Behavioral and Psychological Symptoms of Dementia in Post-stroke Vascular Cognitive Impairment. 2020;(Icosteerr 2018):553-6.

5. Dagonnier M, Donnan GA, Davis SM, Dewey HM, Howells DW. Acute Stroke Biomarkers: Are We There Yet? 2021;12(February):1-16.

6. Makkar M, Arumugam N, Midha D. Challenges Experienced by Physiotherapists in Rehabilitation of Individuals With Poststroke Hemineglect: A Review Study. J Stroke Med. 2020;3(1):10-3.

7. Xu J, He X, Li Q, Liu J, Zhuang M. Higher Platelet-to-Lymphocyte Ratio Is Associated With Worse Outcomes After Intravenous Thrombolysis in Acute Ischaemic Stroke. 2019;10(November):1-8.

8. Kömürcü HF, Gözke E, Doğan Ak P, Kalyoncu Aslan I, Salt I, Özgenç Bi, er Çİ. Changes in neutrophil, lymphocyte, platelet ratios and their relationship with NIHSS after rtPA and/or thrombectomy in ischemic stroke. J Stroke Cerebrovasc Dis. 2020; 29(8).

9. Zheng M, Chen S, Zhu Y, Gu X. Mean platelet volume: a new predictor of ischaemic stroke risk in patients with nonvalvular atrial fibrillation. 2020;1-7.

10. Jr HPA. Classification of Subtypes of Ischemic Stroke History of the Trial of Org 10172 in Acute Stroke Treatment Classification. 2015;

11. Sadeghi F, Kovács S, Zsóri KS, Csiki Z, Bereczky Z, Shemirani AH. Platelet count and mean volume in acute stroke: a systematic review and meta-analysis Platelet count and mean volume in acute stroke: a systematic review and. Platelets. 2019;00(00):1-9. DOI: 10.1080/09537104.2019.1680826

12. Pandian JD, Arora R, Kaur P, Sharma D, Vishwambaran DK, Arima H. Mirror therapy in unilateral neglect after stroke 
Fani Nanda Sihanto et.al. High platelet lymphocyte ratio as predictor of bad outcome in patient with acute ischemic stroke.

(MUST trial): A randomized controlled trial. Neurology. 2014;83(11):1012-7.

13. Papanagiotou P, White CJ. Endovascular reperfusion strategies for acute stroke. JACC Cardiovasc Interv. 2016;9(4):307-17.

14. Walter Johnson, Oyere Onuma, Mayowa Owolabi et al. Stroke: a global response is needed. Bull World Health Organ. 2016 Sep 1;94(9):634-634A.

doi: 10.2471/BLT.16.181636.

15. Sachdev PS, Lipnicki DM, Crawford JD, Brodaty H. The Vascular Behavioral and Cognitive Disorders criteria for vascular cognitive disorders: a validation study. Eur J Neurol. 2019;26(9):1161-7.

16. Lu C, Gao P, Yang Y, Chen X, Wang L, Yu $\mathrm{D}$, et al. Prognostic evaluation of platelet to lymphocyte ratio in patients with colorectal cancer. Oncotarget. 2017;8(49):86287-95.
17. Tsivgoulis G, Palaiodimou L, Katsanos AH, Caso V, Köhrmann M, Molina C, et al. Neurological manifestations and implications of COVID-19 pandemic. Ther Adv Neurol Disord. 2020;13:1-14.

18. Lei T, Ye Y, Zhu X, Smerin D, Gu L, Xiong $\mathrm{X}$, et al. The immune response of $\mathrm{T}$ cells and therapeutic targets related to regulating the levels of $\mathrm{T}$ helper cells after ischaemic stroke. 2021;1-18.

How to cite this article: Sihanto FN, Bahrudin M, Suharto. High platelet lymphocyte ratio as predictor of bad outcome in patient with acute ischemic stroke. International Journal of Research and Review. 2021; 8(10): 277-281. DOI: https://doi.org/10.52403/ijrr.20211037 